\title{
Assurance et Information Imparfaite
}

\author{
par Claude Henry *
}

On considère, dans ces quelques pages, une des idées essentielles présentées à la section $8 \mathrm{du}$ texte du Professeur $\mathrm{K}$. Borch : "Thus in the model presented by Rothschild and Stiglitz it is doubtful if a competitive equilibrium exists, and if it exists it may not be Pareto optimal » (page 27). Et encore : "There will however still be difficulties about the existence of a competitive equilibrium, and about the Pareto optimality of the equilibria 》 (page 28), l'adverbe «still» se référant à l'offre par l'assureur de deux contrats $\left(c_{1}, P_{1}\right)$ et $\left(c_{2}, P_{2}\right)$, où $c_{i}(i=1,2)$ est la compensation offerte moyennant la prime $P_{i}(i=1,2)$. Rothschild et Stiglitz ont montré qu'alors le seul équilibre possible est constitué des deux types de contrats suivants:

$$
c_{1}=x, \quad P_{1}=\pi_{B}
$$

que choisissent les mauvais ( $«$ bad ») risques,

$$
c_{2}=\gamma, \quad P_{2}=\pi_{G}
$$

que choisissent les bons ( good») risques, sachant que $\gamma$ est déterminé par la condition :

$$
u\left(W-x \pi_{B}\right)=\left(1-\pi_{B}\right) u\left(W-\gamma \pi_{G}\right)+\pi_{B} u\left(W-x+\gamma-\gamma \pi_{G}\right) .
$$

La condition (1) exprime qu'est offerte aux bons risques la compensation maximum qui n'induise pas les mauvais risques à choisir le second plutôt que le premier type de contrat offert.

Nous ne réexaminons pas ici les conditions sous lesquelles $\left\{\left(x, \pi_{B}\right),\left(\gamma, \pi_{G}\right)\right\}$ est réellement un équilibre, dont plusieurs concepts ont d'ailleurs été proposés; mais nous nous intéressons aux questions suivantes :

* Professeur à l'Ecole Polytechnique, Paris. 
1. Est-il avantageux au sens de Pareto, c'est-à-dire pour tous les souscripteurs d'assurance, que soient offerts des contrats garantissant l'équilibre financier global de l'assureur, mais dont un type est bénéficiaire alors que l'autre est déficitaire? Chacun des deux types de contrats $\left(x, \pi_{B}\right)$ et $\left(\gamma, \pi_{f}\right)$ est par contre financièrement équilibré.

2. Désignant par $\lambda_{B}$ et $\lambda_{G}$ (avec $\lambda_{B}+\lambda_{G}=1$ ) les proportions respectives de mauvais et bons risques dans la population totale des souscripteurs; par $\mu_{B}$ et $\mu_{G}$ (avec $\mu_{B}+\mu_{G}=1, \mu_{B}<\lambda_{B}$ ) les proportions correspondantes dans une souspopulation délimitée à partir d'un critère vérifiable et non manipulable (par exemple une appartenance professionnelle telle que fonctionnaire), est-il avantageux au sens de Pareto que soient offerts aux souscripteurs appartenant à cette sous-population des contrats qui leur soient réservés et réalisent leur propre équilibre financier global? Autrement dit, peut-on pratiquer pareille discrimination sans léser les autres souscripteurs?

Si la réponse à la première question est positive, il est évident qu'alors la réponse à la seconde est négative. Que la réponse à la première question soit positive signifie en effet qu'il convient que les bons risques subventionnent les mauvais; discriminer, comme l'envisage la seconde question, en faveur d'une sous-population comportant une proportion plus élevée de bons risques, dégrade alors les conditions d'équilibre financier global pour l'ensemble des contrats offerts aux autres souscripteurs.

Pour répondre à la première question, considérons tous les contrats $\left(c_{1}, P_{1}\right)$, avec $P_{1}<\pi_{B}$, situés sur une même courbe d'indifférence d'un mauvais risque; que cette courbe soit définie à partir d'une fonction d'utilité ayant la forme de Von NeumannMorgenstern

$$
\left(1-\pi_{B}\right) u\left(W-c_{1} P_{1}\right)+\pi_{B} u\left(W-x+c_{1}-c_{1} P_{1}\right)
$$

implique que le moins déficitaire des contrats $\left(c_{1}, P_{1}\right)$ considérés comporte la compensation complète $c_{1}=x$. L'assureur peut alors offrir un second type de contrat $\left(c_{2}, P_{2}\right)$, qui soit choisi par les bons risques, tel que

$$
u\left(W-x P_{1}\right)=\left(1-\pi_{B}\right) u\left(W-c_{2} P_{2}\right)+\pi_{B} u\left(W-x+c_{2}-c_{2} P_{2}\right)
$$

et

$$
\lambda_{B} x\left(\pi_{B}-P_{1}\right)=\lambda_{G} c_{2}\left(P_{2}-\pi_{G}\right)
$$

La condition (3) est l'analogue de (1), à laquelle elle se ramène pour $P_{1}=\pi_{B}$, tandis que la condition (4) traduit l'équilibre financier global de l'assureur, $P_{1}$ variant de $\pi_{B}$ à

$$
\frac{\lambda_{B} \pi_{B}+\lambda_{G} \pi_{G}}{\lambda_{B}+\lambda_{G}} .
$$


En éliminant $P_{1}$ entre (3) et (4), on obtient la condition sur les contrats compatibles à la fois avec des contrats choisis par les mauvais risques et plus favorables pour ceux-ci que $\left(x, \pi_{B}\right)$, et avec l'équilibre global de l'assureur :

(5) $u\left(W-x \pi_{B}+\frac{\lambda_{G}}{\lambda_{B}} c_{2}\left(P_{2}-\pi_{G}\right)\right)=\left(1-\pi_{B}\right) u\left(W-c_{2} P_{2}\right)+\pi_{B} u\left(W-x+c_{2}-c_{2} P_{2}\right)$.

La première question peut maintenant être reformulée dans les termes suivants : existe-t-il au moins un contrat $\left(c_{2}, P_{2}\right)$, satisfaisant la condition (5), qui soit plus avantageux pour les bons risques que $\left(\gamma, \pi_{G}\right)$ ? Des calculs sans malice mais un peu lourds fournissent la réponse: oui, à condition que la proportion $\lambda_{G}$ de bons risques soit assez grande ; plus précisément à condition d'avoir :

$$
\frac{\lambda_{G}}{\lambda_{B}}>k \frac{\left(1-\pi_{G}\right) \pi_{B}-\left(1-\pi_{B}\right) \pi_{G}}{\pi_{G}\left(1-\pi_{G}\right)}
$$

le facteur positif $k$ s'écrivant

$$
k=\frac{u^{\prime}\left(W-\gamma \pi_{G}\right) u^{\prime}\left(W-x+\gamma-\gamma \pi_{G}\right)}{u^{\prime}\left(W-\pi_{B} x\right)\left(u^{\prime}\left(W-x+\gamma-\gamma \pi_{G}\right)-u^{\prime}\left(W-\gamma \pi_{G}\right)\right)} .
$$

Si par contre on a

(8)

$$
\frac{\lambda_{G}}{\lambda_{B}} \leqslant k \frac{\left(1-\pi_{G}\right) \pi_{B}-\left(1-\pi_{B}\right) \pi_{G}}{\pi_{G}\left(1-\pi_{G}\right)}
$$

mais aussi

$$
\frac{\mu_{G}}{\mu_{B}}>k \frac{\left(1-\pi_{G}\right) \pi_{B}-\left(1-\pi_{B}\right) \pi_{G}}{\pi_{G}\left(1-\pi_{G}\right)}
$$

alors c'est à la seconde question que la réponse est positive : une offre discriminatoire en faveur de la sous-population caractérisée par les proportions $\mu_{G}$ et $\mu_{B}$ est avantageuse au sens de Pareto. 